\title{
Data envelopment analysis with common weights: the weight restriction approach
}

\author{
Azam Pourhabib Yekta ${ }^{1} \cdot$ Sohrab Kordrostami ${ }^{1} \cdot$ Alireza Amirteimoori $^{2} \cdot$ Reza Kazemi Matin $^{3}$
}

Received: 13 February 2018/Accepted: 10 August 2018/Published online: 28 August 2018

(C) The Author(s) 2018

\begin{abstract}
The conventional data envelopment analysis suggests each decision-making unit selecting its most desirable weight. Applying these weights lets the units achieve their maximum performance. But, the performance of different units is achieved with different sets of weights. So, comparison and ranking of units on a common basis seems such an impossible challenge. However, the flexibility in choosing weights will make more than one efficient unit to be claimed as an efficient unit. In order to resolve these shortcomings, this paper proposes a method that only one common set of weights is obtained through this method. Toward this end, firstly, the efficiency of each unit is calculated, and then, the units are ranked by the efficiency scores earned from common weights. The weight restriction approach here not only generates positive weights but also prevents weights dissimilarity. The production of strictly positive weights through the proposed model makes it possible that no input and output variables are ignored.
\end{abstract}

Keywords Data envelopment analysis · Efficiency · Common set of weight · Weight dissimilarity · Weight restriction

\section{Introduction}

Data envelopment analysis (DEA) is a powerful mathematical programming method that measures the relative performance of units with multiple inputs and outputs. The conventional DEA models assess the best possible relative efficiencies of decision-making units (DMUs). In DEA standard programming, we would like to obtain a measure of the ratio of summation of all weighted outputs to the summation of all weighted inputs. The optimal input and output weights are obtained by solving the mathematical programming such that the efficiency of underevaluated unit is maximized. Although the objective function maximizes efficiency, it is subjected to the constraint that all efficiency measures must be smaller than or equal to one.

Sohrab Kordrostami

sohrabkordrostami@gmail.com

1 Department of Mathematics, Lahijan Branch, Islamic Azad University, Lahijan, Iran

2 Department of Applied Mathematics, Rasht Branch, Islamic Azad University, Rasht, Iran

3 Department of Applied Mathematics, Karaj Branch, Islamic Azad University, Karaj, Iran
Because of the flexibility in choosing input and output's weights, they always have more than one efficient unit. In order to solve this problem, many researchers proposed some methods for ranking DMUs. Generally, the ranking methods are classified into three groups. The first group of ranking methods is super-efficiency method which was introduced by Anderson and Peterson [3]. In this methodology, the unit under evaluation is deleted from the reference set. Infeasibility and instability are two fundamental problems of the super-efficiency method, and due to these problems; the application of this method has been restricted. The super-efficiency method was expanded by many researchers. Alternative researches can be found in Amirteimoori and Kordrostami [1] and [2], Chen [6], Xue and Harker [27], Li et al. [11], and Seiford and Zhu [21]. The second group of ranking method was the cross-efficiency technique which was first developed by Sexton et al. [22]. There are two stages in cross-efficiency method. First, the optimal weights of the unit under evaluation with conventional Charnes et al. [5] (CCR) model are obtained. This stage is called self-evaluation. Then, by applying the optimal weights of self-evaluation stage, other DMUs will be evaluated. This stage is called peer evaluation. Then, cross-efficiencies are obtained by the optimal weights 
selected by all DMUs in the peer evaluation stage. Finally, the average of the cross-efficiencies of each DMU is called cross-efficiency score. This ranking method has many advantages, and it also faces some problems. The main problem of this method is the possibility of alternative optimal solutions for weights in the first stage of evaluation which may cause different cross-efficiency scores. Consequently, different ranking for units will be at hand. This subject has been developed by many researchers like Doyle and Green [8], Dimitrov and Sutton [7], Obata and Ishii [16], Liu and Peng [13], and Liang et al. [12]. The third group of ranking method is common weights. This method discusses the weights which are selected for evaluating and ranking DMUs. As far as we are aware, conventional DEA models let each DMU choose the most desirable weights in order to achieve their own maximum performance. This flexibility in selecting input and output weights often leads to some problems. Common weights have been suggested instead of variable weights to decrease the flexibility in selecting input and output weights. Using common weights makes it possible to discriminate units on a common basis. Many approaches have been proposed to achieve a common set of weights. For example, refer to Roll et al. [20], Roll and Golany [19], Liu and Peng [13], Kao and Hung [10], Wang et al. [25] and Hatami-Marbini et al. [9] and Noura et al. [15] and Ramezani-Tarkhorani et al. [17] and Ramón et al. [18] and Wu et al. [26].

In this paper, a weight restriction approach has been proposed to search one common set of weights. This common set of weights is applied to evaluate the efficiency of each DMU and then rank the DMUs with these obtained efficiency scores. The proposed weight restriction approach generates positive weights and, at the same time, prevents weights dissimilarity. In addition, it reduces efficiency scores and number of efficient units. The modern weight restriction approach is expressed in two different schemes. Firstly, the input/output weights are conjointly restricted. Then, this view is extended, and the input/output weights are restricted individually. Above all, suggested weight restriction approach does not need initial information on the input and output weights.

The rest of the paper has the following order. The next section will present the traditional DEA method. "Weight restriction approach" section represents alternative weight restriction approach in the DEA literature, measures units' efficiency, and then ranks DMUs, including two different weight restriction schemes. "Numerical examples" section gives two numerical examples, and conclusions are offered in section "Conclusions."

\section{Preliminaries}

Suppose that there is a set of $\mathrm{n}$ DMUs, and each $\mathrm{DMU}_{j}$ $(j=1,2, \ldots, n)$ generates $s$ different outputs using $m$ different inputs which are denoted as $x_{i j}(i=1,2, \ldots, m)$ and $y_{r j}(r=1,2, \ldots, s)$, respectively. For any $\mathrm{DMU}_{o}$ $(o=1,2, \ldots, n)$, the efficiency score is computed by the following model called CCR model.

$$
\begin{aligned}
& \operatorname{Max} \operatorname{Eff}_{o}=\frac{\sum_{r=1}^{s} u_{r} y_{r o}}{\sum_{i=1}^{m} v_{i} x_{i o}} \\
& \text { s.t. } \quad \sum_{r=1}^{s} u_{r} y_{r j}-\sum_{i=1}^{m} v_{i} x_{i j} \leq 0, \quad j=1, \ldots, n, \\
& \\
& u_{r}, v_{i} \geq 0, \quad \text { for all } r, i .
\end{aligned}
$$

If the optimal value of the objective function for $\mathrm{DMU}_{o}$ is equal to one, then the $\mathrm{DMU}_{o}$ is efficient; otherwise, it is inefficient. The standard CCR model has the unit invariant property that is organized by Lovell and Pastor [14]. This property makes it possible to normalize the weights. Thus, there is a scale of data that causes model (1) to be equivalent to the following model.

$$
\operatorname{Max}_{\operatorname{Eff}}=\frac{\sum_{r=1}^{s} u_{r} y_{r o}}{\sum_{i=1}^{m} v_{i} x_{i o}}
$$

s.t.

$$
\begin{aligned}
& \sum_{r=1}^{s} u_{r} y_{r j}-\sum_{i=1}^{m} v_{i} x_{i j} \leq 0, \quad j=1, \ldots, n, \\
& 0 \leq u_{r} \leq 1, \quad r=1, \ldots, s \\
& 0 \leq v_{i} \leq 1, \quad i=1, \ldots, m .
\end{aligned}
$$

It can easily be shown that models (1) and (2) are equivalent. It suffices to show this to assume that $u_{r}{ }^{*}$ and $v_{i}^{*}(i=1, \ldots, m, r=1, \ldots, s)$ are optimal solutions to model (1), and we suppose that $\phi$ is maximum of $u_{r}^{*}$ and $v_{i}^{*}(i=1, \ldots, m, r=1, \ldots, s)$. Then, we divide the input/ output weights by $\phi$. The obtained answer gives a feasible solution to model (2).

Comparing both models, it is found that the above models are the same, but the nonnegative variables in model (1) have been replaced with the bounded variables in model (2). Model (2) assists in applying a modified CCR model that prevents dissimilar weights. On the other hand, model (1) allows each DMU to select the best weights in order to maximize its performance score. This subject is faced with some problems. For example, the performance of different units will be achieved by different set of weights. So, the comparison and ranking of units will not be on the same base. Additionally, because of the flexibility in choosing input and output weights, we always have more than one efficient unit. This would lead us to the case that all DMUs cannot be discriminated. 


\section{Weight restriction approach}

In this section, our weight restriction approach is handled in two different schemes. First, we introduced a process that does not distinguish between the selected input and output weights. Then, this idea is developed, and the input/ output weights are restricted individually.

\section{Weight restriction approach with restricting input/output weights conjointly}

In order to assess one common set of weight with no difference in input/output weights, the following model is proposed. The model allows selecting common weights through conjointly restricting the input and output weights with a common bound. What is more, a common bound is employed for input/output weights. The model is represented in the following format:

$\operatorname{Min} \frac{\sum_{j=1}^{n} d_{j}}{\alpha}$

S.t. $\sum_{r=1}^{s} u_{r} y_{r j}-\sum_{i=1}^{m} v_{i} x_{i j}+d_{j}=0, \quad j=1, \ldots, n \quad(3-1)$

$\alpha \leq v_{i} \leq 1, \quad i=1, \ldots, m$

$\alpha \leq u_{r} \leq 1, \quad r=1, \ldots, s$

$u_{r}, v_{i}, \alpha \geq 0$ for all $i$ and $r$.

As constraint (3-1) shows, the variable $d_{j}$ is denoted as deviation variable or slack variable for each unit. Also, $\alpha$ shows lower bound for input and output weights. The constraints (3-2) and (3-3) force all these multipliers to vary between two bounds: the lower bound $\alpha$ and the upper bound 1 . The objective function minimizes the summation of deviation variable, i.e., $\sum_{j=1}^{n} d_{j}$, and maximizes $\alpha$ simultaneously. According to the concept of slack variable in the DEA literature, the proposed objective function with decreasing $\sum_{j=1}^{n} d_{j}$ tries to minimize the summation of deviations of all DMUs and at the same time, with increasing $\alpha$, attempts to search a positive lower bound for common set of weights among all feasible multipliers. Thus, the proposed weight restriction approach generates positive weights and prevents weight dissimilarity. Notably, there is no need to have prior information about the input and output weights for determining the bound in this proposed approach. The important point is that the proposed model, which restricts the weights jointly, gives fewer efficient units compared with conventional CCR model.

As it is clear, because of construction of model's objective function, model (3) is nonlinear. Furthermore, the model can turn into linear programming with the Charnes and Cooper [4] transformation.

The following theorem proves that the proposed weight restriction approach is feasible and generates positive weights.

Theorem 1 Model (3) is feasible and it generates positive weights in optimality.

Proof Assume that in evaluating $\mathrm{DMU}_{o}, \mathrm{DMU}_{d}$ is a reference unit with the CCR model. Thus, in evaluating $\mathrm{DMU}_{d}$ with model (2), we have: $u_{r}^{d}>0$ and $v_{i}^{d}>0$ $(i=1, \ldots, m, r=1, \ldots, s)$. So, it can be concluded that $u_{r}^{d}, v_{i}^{d}, \alpha=\operatorname{Min}_{i, r}\left\{u_{r}^{d}, v_{i}^{d}\right\}$ is a feasible solution for evaluating $\mathrm{DMU}_{o}$ by model (3) with $\alpha>0$.

\section{Weight restriction approach with restricting input/output weight individually}

The proposed weight restriction approach is now expanded to a more general case. In this subsection, a linear formulation is also proposed to determine positive optimal weight for common set of weights among all feasible multipliers. For this purpose, the input/output weights are limited individually. The following formulation not only determines positive common set of weights but also prevents weights dissimilarity, simultaneously. The model is formatted as:

$\operatorname{Min} \frac{\sum_{j=1}^{n} d_{j}}{\alpha}$

S.t. $\sum_{r=1}^{s} u_{r} y_{r j}-\sum_{i=1}^{m} v_{i} x_{i j}+d_{j}=0, \quad j=1, \ldots, n \quad(4-1)$

$w_{I} \leq v_{i} \leq 1, \quad i=1, \ldots, m$

$w_{o} \leq u_{r} \leq 1, \quad r=1, \ldots, s$

$w_{I} \geq \alpha$

$w_{o} \geq \alpha$

$w_{I}, w_{o}, u_{r}, v_{i}, \alpha \geq 0$, for all $r, i$.

The variable $d_{j}$ is the deviation variable or slack variable for each DMU in the constraint (4-1). The constraint (4-2) forces all input multiples to vary between the bounds $w_{I}$ and 1 and also forces all output multiples to vary in-between the bounds $w_{o}$ and 1 in the constraint (4-3). The constraints (4-4) and (4-5) limit lower bound of input and output weights to the positive variable $\alpha$. Similar to the idea behind model (3), in this model (4), with minimizing $\sum_{j=1}^{n} d_{j}$ and maximizing $\alpha$ in the objective function, one common set of weights is searched. As it is mentioned before, the objective function focuses on minimizing the summation of deviation of all DMUs through $\sum_{j=1}^{n} d_{j}$, and 
at the same time, with maximizing lower bound of input and output weights in the fraction's denominator, the model operates by decreasing the distance between $w_{I}$ and $w_{o}$ with the corresponding upper bounds. Above all, the model looks for the weights with the least dissimilarity.

As it is clear, model (4) is a nonlinear mathematical programming. The optimal answer to model (4) is obtained by the following change of variables:

$$
\begin{aligned}
r & =\frac{1}{\alpha}, u_{r}^{\sim}=r u_{r}, v_{i}^{\sim}=r v_{i}, d_{j}^{\sim}=r d_{j}, w_{I}^{\sim} \\
& =r w_{I}, \quad w_{0}^{\sim}=r w_{0}
\end{aligned}
$$

The above transformation leads to the following linear programming problem:

$$
\begin{array}{ll}
\min & \sum_{j=1}^{n} d_{j}^{\sim} \\
\text { s.t. } \quad \sum_{r=1}^{s} u_{r}^{\sim} y_{r j}-\sum_{i=1}^{m} u_{r}^{\sim} x_{i j}+d_{j}^{\sim}=0, \quad j=1, \ldots, n \\
w_{I}^{\sim} \leq v_{i}^{\sim} \leq r, \quad i=1, \ldots, m \\
w_{0}^{\sim} \leq u_{r}^{\sim} \leq r, \quad r=1, \ldots, s \\
w_{I}^{\sim} \geq 1 \\
w_{0}^{\sim} \geq 1 \\
w_{I}^{\sim}, w_{0}^{\sim}, u_{r}^{\sim}, v_{i}^{\sim}, r \geq 0, \quad \forall i, \forall r
\end{array}
$$

Model (5) is the linear format of model (4). The following theorem proves that the above-proposed model (4) is always feasible and generates positive weights.

Theorem 2 Model (4) is feasible, and it generates positive weights in optimality.

Proof The proof of this theorem is similar to Theorem 1.

\section{Numerical examples}

In this section, the proposed weight restriction approach is compared with Kao and Hung's [10] models. Kao and Hung's [10] suggested three models using different distance function to produce common weights. Refer to Kao and Hung paper [10] for more details.

\section{Example 1}

In that example, there are 17 forest areas in Taiwan with four inputs and three outputs from which Taiwan's forest areas data are chosen from Kao and Hung [10]. The data set including four inputs: budget, initial stocking, labor, and land and three outputs: main product, soil conservation, and recreation are considered for measuring the efficiency. The input/output data sets are listed in Table 1.
Table 1 Data set

\begin{tabular}{llrlllll}
\hline DMU & \multicolumn{1}{l}{$x_{1}$} & \multicolumn{1}{c}{$x_{2}$} & $x_{3}$ & $x_{4}$ & $y_{1}$ & $y_{2}$ & $y_{3}$ \\
\hline 1 & 51.6 & 11.2 & 49.2 & 33.2 & 40.4 & 14.8 & 3166.7 \\
2 & 85.7 & 123.9 & 55.1 & 108.4 & 43.5 & 173.5 & 6.4 \\
3 & 66.6 & 104.1 & 257 & 13.6 & 139.7 & 115.9 & 0 \\
4 & 27.8 & 107.6 & 14 & 146.4 & 25.4 & 131.7 & 0 \\
5 & 51.2 & 117.5 & 32 & 84.5 & 46.2 & 144.9 & 0 \\
6 & 36.0 & 193.3 & 59.5 & 8.2 & 46.8 & 190.8 & 822.9 \\
7 & 25.8 & 105.8 & 9.5 & 227.2 & 19.4 & 120 & 0 \\
8 & 123 & 82.4 & 87.3 & 98.8 & 43.3 & 125.8 & 404.6 \\
9 & 61.9 & 99.7 & 33 & 86.3 & 45.4 & 79.6 & 1252.6 \\
10 & 80.3 & 104.6 & 53.3 & 79 & 27.2 & 132.4 & 42.6 \\
11 & 205.9 & 183.4 & 144.1 & 59.6 & 14 & 196.2 & 16.1 \\
12 & 82.0 & 104.9 & 46.5 & 127.2 & 44.8 & 108.5 & 0 \\
13 & 202.2 & 187.7 & 149.3 & 93.6 & 44.9 & 184.7 & 0 \\
14 & 67.5 & 82.8 & 44.3 & 60.8 & 26 & 85 & 23.9 \\
15 & 72.6 & 132.7 & 44.6 & 173.4 & 5.5 & 135.6 & 24.1 \\
16 & 84.8 & 104.2 & 159.1 & 171.1 & 11.5 & 110.2 & 49 \\
17 & 71.7 & 88.1 & 69.1 & 123.1 & 44.8 & 74.5 & 6.1 \\
\hline
\end{tabular}

In order to shed a light on strength of the proposed model, the proposed weight restriction model (4) and mentioned Kao and Hung [10] approach are applied on data from Table 1. The results are recorded in Table 2.

The last row in Table 2 depicts the results of our proposed method (model 4), and the rest of the rows shows three different results obtained from Kao and Hung [10] method. Table 3 shows the efficiency scores of data set from Table 1. The efficiency scores are calculated by CCR model, Kao and Hung [10] models, and the weight restriction approach proposed in this paper, respectively. The notation Eff ${ }^{\text {new }}$ is drawn for the proposed common set of weights approach.

As mentioned before, Table 3 shows the efficiency score of 17 DMUs calculated by five models. The second column of Table 3 gives the CCR efficiency scores. It is clear that there are nine efficient DMUs. However, it is difficult to put rank for efficient units. Additionally, each unit has been evaluated by its own weights. In order to resolve these problems, Kao and Hung [10] suggested three common weight DEA models. These three models are used to calculate the efficiency of these 17 DMUs. The results are recorded in the third, fourth, and fifth columns of Table 3. The efficiency scores of the weight restriction approach proposed in this paper are shown in the last column of Table 3. It is clear that the number of efficient units is dropped from nine to three in the proposed method. Another strength of the proposed approach turns back to efficiency scores. It is clear that the proposed model (4) gives the least efficiency scores compared with other 
Table 2 Results of comparison

\begin{tabular}{|c|c|c|c|c|c|c|c|}
\hline Model & Budget & Initial stocking & Labor & Land & Main product & Soil conservation & Recreation \\
\hline$P=1$ & 1.097540 & 2.645978 & 0.000100 & 0.000100 & 0.608528 & 2.274926 & 0.008799 \\
\hline$P=2$ & 1.227655 & 2.523662 & 0.000100 & 0.150043 & 0.153914 & 2.368929 & 0.012841 \\
\hline$P=\propto$ & 0.062274 & 1.629378 & 0.838507 & 0.329658 & 0.830676 & 1.455490 & 0.005851 \\
\hline Model (4) & 0.478000 & 0.703234 & 0.920025 & 0.110012 & 0.020782 & 1.00000 & 0.020782 \\
\hline
\end{tabular}

Table 3 Efficiency scores and the associated ranking (in parentheses) calculated by CCR ratio model and different methods of common weights

\begin{tabular}{llllll}
\hline DMUs & CCR & $P=1$ & $P=2$ & $P=\propto$ & Eff $^{\text {new }}$ \\
\hline 1 & $1(1)$ & $1(1)$ & $1(1)$ & $1(1)$ & $0.9998(4)$ \\
2 & $1(1)$ & $1(1)$ & $1(1)$ & $1(1)$ & $0.9175(6)$ \\
3 & $1(1)$ & $1(1)$ & $0.998(3)$ & $0.723(11)$ & $0.3463(17)$ \\
4 & $1(1)$ & $1(1)$ & $0.992(4)$ & $0.898(4)$ & $1(1)$ \\
5 & $1(1)$ & $0.974(5)$ & $0.986(5)$ & $1(1)$ & $1(1)$ \\
6 & $1(1)$ & $0.852(9)$ & $0.912(6)$ & $0.869(7)$ & $0.988(5)$ \\
7 & $1(1)$ & $0.924(6)$ & $0.884(7)$ & $0.743(9)$ & $1(1)$ \\
8 & $1(1)$ & $0.895(7)$ & $0.870(9)$ & $0.893(5)$ & $0.6497(10)$ \\
9 & $1(1)$ & $0.661(14)$ & $0.669(14)$ & $0.723(12)$ & $0.723(8)$ \\
10 & $0.940(10)$ & $0.872(8)$ & $0.876(8)$ & $0.876(6)$ & $0.7891(7)$ \\
11 & $0.934(11)$ & $0.639(15)$ & $0.651(15)$ & $0.657(13)$ & $0.5370(13)$ \\
12 & $0.829(12)$ & $0.745(10)$ & $0.728(10)$ & $0.759(8)$ & $0.6445(11)$ \\
13 & $0.799(13)$ & $0.622(17)$ & $0.626(16)$ & $0.645(14)$ & $0.4933(14)$ \\
14 & $0.773(14)$ & $0.714(12)$ & $0.714(12)$ & $0.740(10)$ & $0.6232(12)$ \\
15 & $0.762(15)$ & $0.724(11)$ & $0.721(11)$ & $0.641(15)$ & $0.7209(9)$ \\
16 & $0.743(16)$ & $0.699(13)$ & $0.681(13)$ & $0.466(17)$ & $0.3994(16)$ \\
17 & $0.687(17)$ & $0.631(16)$ & $0.606(17)$ & $0.590(16)$ & $0.4357(15)$ \\
Average & 0.910 & 0.821 & 0.818 & 0.788 & 0.7224 \\
\hline & & & & &
\end{tabular}

methods. As the last row of Table 3 records, the average of efficiency in model (4) is 0.724 , while the average efficiency is recorded as 0.910 in CCR model and 0.818 in Kao and Hung [10] first model with $P=1$. For the rest of the two models of Kao and Hung [10] with $P=2$ and $P=\propto$, the average efficiency score is drawn as 0.818 and 0.788 , respectively. The results show that the average of efficiency scores in our approach is less than that in other models. Therefore, our weight restriction approach along with a common set of weights not only produces the strictly positive weights but also prevents dissimilar weights. In comparison with Kao and Hung approach [10], our weight restriction approach, models (3) and (4), has another strength, that is, there is no need to calculate the CCR efficiency score. This clue makes the proposed method more applicable than the existing Kao and Hung [10] method.
Table 4 Data set for twelve flexible manufacturing systems

\begin{tabular}{lrlllll}
\hline FMS & \multicolumn{1}{l}{$x_{1}$} & $x_{2}$ & $y_{1}$ & $y_{2}$ & $y_{3}$ & $y_{4}$ \\
\hline 1 & 17.02 & 5 & 42 & 45.3 & 14.2 & 30.1 \\
2 & 16.46 & 4.5 & 39 & 40.1 & 13 & 29.8 \\
3 & 11.76 & 6 & 26 & 39.6 & 13.8 & 24.5 \\
4 & 10.52 & 4 & 22 & 36 & 11.3 & 25 \\
5 & 9.50 & 3.8 & 21 & 34.2 & 12 & 20.4 \\
6 & 4.79 & 5.4 & 10 & 20.1 & 5 & 16.5 \\
7 & 6.21 & 6.2 & 14 & 26.5 & 7 & 19.7 \\
8 & 11.12 & 6 & 25 & 35.9 & 9 & 24.7 \\
9 & 3.67 & 8 & 4 & 17.4 & 0.1 & 18.1 \\
10 & 8.93 & 7 & 16 & 34.3 & 6.5 & 20.6 \\
11 & 17.74 & 7.1 & 43 & 45.6 & 14 & 31.1 \\
12 & 14.85 & 6.2 & 27 & 38.7 & 13.8 & 25.4 \\
\hline
\end{tabular}

\section{Example 2}

This example includes 12 flexible manufacturing systems which are shown in Table 4 . These data are chosen from 
Table 5 Common weights derived by different models

\begin{tabular}{lllllll}
\hline Model & $I_{1}$ & $I_{2}$ & $O_{1}$ & $O_{2}$ & $O_{3}$ & $O_{4}$ \\
\hline$P=1$ & 0.076680 & 0.026510 & 0.021330 & 0.004024 & 0 & 0.011944 \\
$P=2$ & 0.928986 & 0.386831 & 0.225722 & 0 & 0 & 0.254175 \\
$P=\propto$ & 0.896384 & 0.415631 & 0.150772 & 0.05559 & 0 & 0.238215 \\
Model(4) & 1.00000 & 0.3280 & 0.1226 & 0.1226 & 0.1226 & 0.1226 \\
\hline
\end{tabular}

Table 6 Efficiency scores calculated by CCR model and different models of common weights

\begin{tabular}{llllll}
\hline DMUs & CCR & $P=1$ & $P=2$ & $P=\propto$ & Eff $^{\text {new }}$ \\
\hline 1 & 1 & 1 & 0.9654 & 0.9111 & 0.8646 \\
2 & 1 & 0.9788 & 0.9616 & 0.9026 & 0.8332 \\
3 & 0.9824 & 0.9488 & 0.9132 & 0.9021 & 0.9001 \\
4 & 1 & 1 & 1 & 1 & 0.9771 \\
5 & 1 & 1 & 0.9641 & 0.9663 & 0.9993 \\
6 & 1 & 0.9624 & 0.9866 & 0.9872 & 0.9611 \\
7 & 1 & 1 & 1 & 1 & 1 \\
8 & 0.9614 & 0.9614 & 0.9423 & 0.9203 & 0.8861 \\
9 & 1 & 0.7528 & 0.8462 & 0.8760 & 0.7713 \\
10 & 0.9536 & 0.8334 & 0.8041 & 0.8295 & 0.8052 \\
11 & 0.9831 & 0.9507 & 0.9160 & 0.8591 & 0.8167 \\
12 & 0.8012 & 0.7943 & 0.7750 & 0.7602 & 0.7600 \\
Average & 0.9734 & 0.9317 & 0.9228 & 0.9095 & 0.8812 \\
\hline
\end{tabular}

Shang and Sueyoshi [23]. The data set includes two inputs: annual operating and depreciation costs, and the floor space requirements of each specific system, and four outputs: the improvement qualitative benefits, work in process, average number of tardy jobs, and average yield.

As before, Kao and Hung [10] and proposed method are performed on this data set. The results are recorded in Table 5. The results of Table 5 show that the third output weight in Kao and Hung models is always zero, while the weight of proposed model for all outputs is strictly positive. (The results of Kao and Hung [10] models are taken from the Sun et al. [24]).

Regarding Table 6, efficiency scores of data set from Table 4 are recorded. The efficiency scores are calculated by CCR model, the compromise solution approach by Kao and Hung [10], and the weight restriction approach proposed in this paper, respectively. The notation $\mathrm{Eff}^{\text {new }}$ is drawn for the proposed common set of weights approach.

It can be seen that proposed restriction approach reduced the number of DEA-efficient DMUs from seven in CCR model to one. So, the proposed weight restriction approach gives fewer efficient units compared with the others models. Regarding the average of efficiencies, reported in the last row of Table 6, the proposed method attains the least value. The average score of efficiency is 0.973 in CCR; $0.931,0.922$ and 0.909 in Kao and Hung model for $P=1$,
$P=2$, and $P=\propto$, respectively; and 0.881 in our weight restriction approach. The results imply that the average of efficiency scores in our approach is less than that in other models. Thus, the proposed weight restriction approach not only leads to strictly positive weights but also prevents dissimilar weights.

\section{Conclusions}

This paper proposed alternative weight restriction approach to generate a common set of weights for all DMUs. Besides, this common set of weights looks suitable for a fair ranking of DMUs. Our proposed model not only generates strictly positive weights but also prevents weights dissimilarity. The production of strictly positive weights by the proposed model makes it possible that no input and output variables are ignored. Furthermore, it reduces the number of efficient units and gives less efficiency score. However, there is no need for initial information on the input and outputs weights in this approach. Admittedly, the proposed method can be extended to other standard DEA models with appropriate modifications. Equally, the proposed weight restriction approach may suffer from existence of alternative optimal solution for common set of weights, which can be investigated in future works.

Acknowledgements The authors are indebted to the anonymous referees for valuable comments and suggestions, which have greatly improved the earlier version of this paper. The authors thank the editor Professor Khosrow Maleknejad for his exceptionally fast services.

Open Access This article is distributed under the terms of the Creative Commons Attribution 4.0 International License (http://creative commons.org/licenses/by/4.0/), which permits unrestricted use, distribution, and reproduction in any medium, provided you give appropriate credit to the original author(s) and the source, provide a link to the Creative Commons license, and indicate if changes were made.

\section{References}

1. Amirteimoori, A., Kordrostami, S.: A Euclidean distance-based measure of efficiency in data envelopment analysis. Optimization 59(7), 985-996 (2010) 
2. Amirteimoori, A., Kordrostami, S.: A distance-based measure of super efficiency in data envelopment analysis: an application to gas companies. J. Glob. Optim. 54(1), 117-128 (2012)

3. Anderson, P., Peterson, N.C.: A procedure for ranking efficient units in data envelopment analysis. Manag. Sci. 39, 1261-1264 (1993)

4. Charnes, A., Cooper, W.W.: Programming with linear fractional. Nav. Res. Logist. Q. 9, 181-196 (1962)

5. Charnes, A., Cooper, W.W., Rhodes, E.: Measuring the efficiency of decision making units. Eur. J. Oper. Res. 2, 429-444 (1978)

6. Chen, Y.: Ranking efficient units in DEA. Omega 32, 213-219 (2004)

7. Dimitrov, S., Sutton, W.: Promoting symmetric weight selection in data envelopment analysis: a penalty function approach. Eur. J. Oper. Res. 200(1), 281-288 (2010)

8. Doyle, J., Green, R.: Efficiency and cross efficiency in DEA: derivations, meaning and the uses. J. Oper. Res. Soc. 45, 567-578 (1994)

9. Hatami-Marbini, A., Tavana, M., Agrell, P.J., Hosseinzadeh Lotfi, H., Ghelej Beigi, Z.: A common-weights DEA model for centralized resource reduction and target setting. Comput. Ind. Eng. 79, 195-203 (2015)

10. Kao, C., Hung, H.T.: Data envelopment analysis with common weights: the compromise solution approach. J. Oper. Res. Soc. 56, 1196-1203 (2005)

11. Li, S., Jahanshaloo, G.R., Khodabakhshi, M.: A super efficiency model for ranking efficient units in data envelopment analysis. Appl. Math. Comput. 184, 638-648 (2007)

12. Liang, L., Wu, J., Cook, W.O., Zhu, J.: Alternative secondary goals in DEA cross-efficiency evaluation. Int. J. Prod. Econ. 113, 1025-1030 (2008)

13. Liu, F.H.F., Peng, H.H.: Ranking of units on the DEA frontier with common weights. Comput. Oper. Res. 35(5), 1624-1637 (2008)

14. Lovell, C.A.K., Pastor, J.T.: Units invariant and translation invariant DEA models. Oper. Res. Lett. 18, 147-151 (1995)

15. Noura, A., Hosseinzadeh Lotfi, F., Hajihosseini, A.: Congestion in DEA under weight restriction using common weights. J. Math. Extens. 1, 121-133 (2016)
16. Obata, T., Ishii, H.: A method for discriminating efficient candidates with ranked voting data. Eur. J. Oper. Res. 151, 233-237 (2003)

17. Ramezani-Tarkhorani, S., Khodabakhshi, M., Mehrabian, S., Nuri-Bahmani, F.: On ranking decision making units with common weights in DEA. Appl. Math. Model. 38, 3890-3896 (2014)

18. Ramón, N., Ruiz, J., Sirvent, I.: Common sets of weights as summaries of DEA profiles of weights: with an application to the ranking of professional tennis players. Expert Syst. Appl. 39, 4882-4889 (2012)

19. Roll, Y., Golany, B.: Alternate methods of treating factor weights in DEA. Omega 21, 99-109 (1993)

20. Roll, Y., Cook, W.D., Golany, B.: Controlling factor weights in data envelopment analysis. IIE Trans. 23, 2-9 (1991)

21. Seiford, L.M., Zhu, J.: Infeasibility of super-efficiency data envelopment analysis. INFOR 37(2), 174-187 (1999)

22. Sexton, T.R., Silkman, R.H., Hogan, A.J.: Data envelopment analysis: critique and extensions. In: Silkman, R.H. (ed.) Measuring Efficiency: An Assessment of Data Envelopment Analysis, pp. 73-105. Jossy-Bass, San Fransisco (1986)

23. Shang, J., Sueyoshi, T.: Theory and methodology-a unified framework for the selection of a flexible manufacturing system. Eur. J. Oper. Res. 85, 297-315 (1995)

24. Sun, Jiasen., Wu, Jie., Gue, Dong.: Performance ranking of units considering ideal and anti-ideal DMU with common weights. Appl. Math. Model. 37, 6301-6310 (2013)

25. Wang, Y.M., Luo, Y., Liang, L.: Ranking decision making units by imposing a minimum weight restriction in data envelopment analysis. J. Comput. Appl. Math. 223(1), 469-484 (2009)

26. Wu, J., Chu, J., Qingyuan, Z., Yongjun, L., Liang, L.: Determining common weights in data envelopment analysis based on the satisfaction degree. J. Oper. Res. Soc. 67, 1446-1458 (2017)

27. Xue, M., Harker, P.T.: Note: Ranking DMUs with infeasible super-efficiency DEA. Eur. J. Oper. Res. 90, 451-460 (2002)

Publisher's Note Springer Nature remains neutral with regard to jurisdictional claims in published maps and institutional affiliations. 